\title{
Time Matched Derivation Indicator
}

National Cancer Institute

\section{Source}

National Cancer Institute. Time Matched Derivation Indicator. NCI Thesaurus. Code C125936.

An indication that data points gathered at the same timepoint will be used in the statistical analysis. 